\title{
A Role for Antimicrobial Stewardship in Clinical Sepsis Pathways: a Prospective Interventional Study
}

Article in Infection Control and Hospital Epidemiology · July 2017

DOI: $10.1017 /$ ice.2017.139

CITATION

1

8 authors, including:

(2.) Suman Adhikari

St George Hospital

7 PUBLICATIONS 17 CITATIONS

SEE PROFILE

(9)

Tomas Jensen

Médecins Sans Frontières, New York / Paris

9 PUBLICATIONS $\mathbf{3 0}$ CITATIONS

SEE PROFILE

\section{READS}

118

Melissa Kelly

Prince of Wales Hospital and Community Health Services 3 PUBLICATIONS 3 CITATIONS

SEE PROFILE

Pam Konecny UNSW Sydney

57 PUBLICATIONS $\quad \mathbf{2 , 5 6 0}$ CITATIONS

SEE PROFILE

Some of the authors of this publication are also working on these related projects:

Mobile technology to support self management of hypertension View project

SEPSIS View project 


\title{
A Role for Antimicrobial Stewardship in Clinical Sepsis Pathways: a Prospective Interventional Study
}

\author{
John Burston, MBBS, FRACP; ${ }^{1,2}$ Suman Adhikari, B Pharm, Grad Dip Clin Pharm; ${ }^{2,3}$ Andrew Hayen, BA(Hons), \\ M. Biostat, PhD; ${ }^{4,5}$ Heather Doolan, BN, MPH; ${ }^{6}$ Melissa L. Kelly, BMed, MPH\&TM; ${ }^{1,2}$ Kathy Fu, MBBS; ${ }^{1,2}$ \\ Tomas O. Jensen, MD, MSc; ${ }^{1,2}$ Pamela Konecny, MBBS, MPH, MD(Res) $)^{1,2}$
}

овлестіve. To evaluate the impact of early infectious diseases (ID) antimicrobial stewardship (AMS) intervention on inpatient sepsis antibiotic management.

DESIGN. Interventional, nonrandomized, controlled study.

Setting. Tertiary-care referral hospital, Sydney, Australia.

Patients. Consecutive, adult, non-intensive care unit (non-ICU) inpatients triggering an institutional clinical sepsis pathway from May to August 2015.

INTERVENTION. All patients reviewed by an ID Fellow within 24 hours of sepsis pathway trigger underwent case review and clinic file documentation of recommendations. Those not reviewed by an ID Fellow were considered controls and received standard sepsis pathway care. The primary outcome was antibiotic appropriateness 48 hours after sepsis trigger.

RESULTS. In total, 164 patients triggered the sepsis pathway: 6 patients were excluded (previous sepsis trigger); 158 patients were eligible; 106 had ID intervention; and 52 were control cases. Of these 158 patients, 91 (58\%) had sepsis, and 15 of these 158 (9.5\%) had severe sepsis. Initial antibiotic appropriateness, assessable in 152 of 158 patients, was appropriate in 80 (53\%) of these 152 patients and inappropriate in 72 (47\%) of these patients. In the intervention arm, $93 \%$ of ID Fellow recommendations were followed or partially followed, including $53 \%$ of cases in which antibiotics were de-escalated. ID Fellow intervention improved antibiotic appropriateness at 48 hours by 24\% (adjusted risk ratio, $1.24 ; 95 \%$ confidence interval, 1.04-1.47; $P=.035)$. The appropriateness agreement among 3 blinded ID staff opinions was $95 \%$. Differences in intervention and control group mortality (13\% vs $17 \%)$ and median length of stay (13 vs 17.5 days) were not statistically significant.

CONCLUSION. Sepsis overdiagnosis and delayed antibiotic optimization may reduce sepsis pathway effectiveness. Early ID AMS improved antibiotic management of non-ICU inpatients with suspected sepsis, predominantly by de-escalation. Further studies are needed to evaluate clinical outcomes.

Infect Control Hosp Epidemiol 2017;1-7

Sepsis represents a major international health challenge with high mortality. ${ }^{1,2}$ Although early antibiotic therapy is likely to reduce mortality in severe sepsis, ${ }^{3}$ recent debate has focused on the importance of antibiotic appropriateness in addition to timing. $^{1,4,5}$ The international 'surviving sepsis' campaign focused on improving outcomes in sepsis. ${ }^{6}$ In New South Wales, Australia, the Clinical Excellence Commission (CEC) introduced statewide clinical sepsis pathways for emergency departments and subsequently for non-intensive care unit (non-ICU) inpatient wards in 2014, as part of a quality improvement program. ${ }^{7}$ The CEC sepsis pathways promote performing blood cultures, measuring serum lactate levels, conducting intravenous fluid resuscitation, and administering antibiotics within 60 minutes of sepsis recognition. ${ }^{7}$ The CEC pathways are based on the Sepsis-2 definitions of sepsis as infection with 2 or more systemic inflammatory response syndrome (SIRS) criteria and of severe sepsis as sepsis causing organ dysfunction. ${ }^{6}$ The CEC inpatient sepsis pathway was implemented in this hospital in October 2014 (referred to hereafter as 'the sepsis pathway') following an intensive preimplementation phase of junior medical officer and nurse education, resource development within the rapid response

Affiliations: 1. Department of Infectious Diseases, Immunology and Sexual Health, St George Hospital, Sydney, Australia; 2. St George and Sutherland Clinical School, Faculty of Medicine, University of New South Wales, Sydney, Australia; 3. Pharmacy Department, St George Hospital, Sydney, Australia; 4. School of Public Health and Community Medicine, University of New South Wales, Sydney, Australia; 5. Faculty of Health, University of Technology, Sydney, Australia; 6. St George Hospital Nursing Administration, Sydney, Australia.

Received February 27, 2017; accepted June 7, 2017

(C) 2017 by The Society for Healthcare Epidemiology of America. All rights reserved. DOI: 10.1017/ice.2017.139 
program, and provision of ward 'sepsis kits' containing otherwise restricted antibiotics.

Preliminary evaluation of our hospital sepsis pathway implementation revealed that half of the patients who triggered the pathway did not have sepsis and were frequently prescribed prolonged broad-spectrum antibiotic courses requiring infectious disease (ID) antimicrobial stewardship (AMS) intervention. Overprescription of broad-spectrum antibiotics was identified as a potential risk to patient safety and a target for intervention. ${ }^{8}$ The aim of this study was to improve antibiotic management for inpatients triggering the sepsis pathway by early ID case review.

\section{METHODS}

\section{Study Design, Setting and Population}

This investigation was an interventional, nonrandomized, controlled study of adult non-ICU inpatients conducted in a 660-bed tertiary-care referral hospital in Sydney, Australia. Consecutive patients who triggered the sepsis pathway between May and August 2015 were enrolled. The intervention was case review within 24 hours of the sepsis pathway trigger, by an infectious diseases advanced trainee (IDAT), an ID fellow equivalent trained in sepsis and AMS management and clinical practice improvement (CPI) methodology. Application of the plan-do-study-act (PDSA) cycle methodology identified IDAT intervention as the most likely to be effective. ${ }^{9}$ Hospitalwide clinical staff education was undertaken prior to implementation, including training videos, intranet resources, and empiric sepsis antibiotic prescribing guide.

The sepsis pathway was triggered when deterioration in a patient's vital signs was suspected to be due to sepsis by either the treating team or by the rapid response medical team. ${ }^{7}$ Specifically, 2 or more of the following vital signs in the context of infection would prompt sepsis pathway trigger: respiratory rate $\leq 10$ or $\geq 25$ respirations per minute, $\mathrm{SpO} 2<95 \%$, systolic blood pressure $<100 \mathrm{mmHg}$, heart rate $\leq 50$ or $\geq 120$ beats per minute, altered consciousness or new onset of confusion, temperature $<35.5^{\circ} \mathrm{C}$ or $>38.5^{\circ} \mathrm{C}^{7}$ The trigger time was recorded as the time of the rapid response call or, if no rapid response was instituted, the time when abnormal vital signs consistent with sepsis were recognized. The treating team generated an electronic alert to notify the IDAT of a sepsis pathway case.

The intervention group received an IDAT review within 24 hours either during that working day or the next day so that timely recommendations for antibiotic management and investigations could be documented as a handwritten entry in the patient file. Written acknowledgement of IDAT review by the treating team was confirmed. A formal ID consulting process involving a senior ID physician was not undertaken unless the treating team requested it. No patients had received ID review prior to enrollment. The control group comprised those patients who triggered the sepsis pathway (ie, generating an electronic alert) but did not receive IDAT intervention due to lack of availability (eg, Saturday trigger or competing clinical priorities) and received standard care from their treating team. The electronic alert contained patient identifying information, sepsis trigger time, ward location and department without clinical case details; these alerts were reviewed consecutively by the IDAT as work schedule permitted. There was no prescreening and, thus, no prioritizing of alerts. Patients were excluded from the study if they had previously triggered the sepsis pathway (Figure 1).

The following data were collected retrospectively from the patient's electronic medical record and paper files following discharge or death: baseline demographics at the time of sepsis trigger, likely source of sepsis, whether blood cultures and serum lactate were obtained, vital signs and laboratory data to determine whether SIRS criteria for sepsis were met and to calculate the APACHE II score for sepsis severity, and microbiology data at both time points. The antibiotics prescribed, dose and time of administration, were recorded for the period immediately prior to, immediately after the alert, and at 48 hours after the pathway trigger.

Antibiotic appropriateness was categorized as 'appropriate,' 'inappropriate' or 'not assessable' according to National Antimicrobial Prescribing Survey (NAPS) criteria. ${ }^{10}$ These standardized criteria are used nationally in periodic pointprevalence surveys. They classify prescriptions as 'appropriate' if consistent with local or national therapeutic guidelines ${ }^{11}$ or directed by microbiological results, with subcategories of 'optimal' and 'adequate.' The 'inappropriate' classification refers to antibiotic prescribing where 'appropriate' criteria are not met, with subcategories of 'suboptimal' and 'inadequate.' Antibiotic appropriateness, was assessed immediately after the sepsis pathway trigger and at 48 hours after the trigger (a recommended pathway review time point). 'Inappropriate' initial antibiotic therapy may be exemplified by an empiric prescription of cefazolin for presumptive line-related sepsis when a patient is colonized with MRSA. 'Appropriate' initial antibiotic therapy of pipericillin/tazobactam plus gentamicin for presumptive severe nosocomial urosepsis would be classified at 48 hours as 'inappropriate' if it was not de-escalated when ampicillin-susceptible Escherichia coli was isolated from the urine and bloodstream. The primary outcome was defined as antibiotic appropriateness at 48 hours post sepsis pathway trigger. Patients classified as unassessable due to missing clinical data at the time of IDAT review were excluded from the appropriateness analysis (Figure 1). Total length of antibiotic therapy was calculated by combining days of parenteral, oral, and discharge prescriptions. Secondary outcomes analyzed were length of therapy, admission to the ICU, formal ID consultations, length of stay (LOS), and in-hospital mortality.

Interventions recommended by the IDAT and the compliance of the treating team were recorded. Compliance was defined as 'followed' if all recommendations were implemented within 48 hours, 'partially followed' if at least 1 but not 
all recommendations were implemented within 48 hours, or 'not followed.'

The first author was responsible for conducting the IDAT intervention. Data retrieved from patient electronic records and paper-based files by a blinded author were assessed separately by 2 blinded senior ID physician coauthors to establish the inter-rater reliability for antibiotic appropriateness, and the diagnoses of sepsis and severe sepsis. The outcome of any disagreement between assessors was determined by a consensus decision between the 2 ID physicians.

\section{Data Analysis}

Dichotomous baseline and outcome measurements between groups was compared using a $\chi^{2}$ or Fisher exact test, as appropriate. For continuous variables, a t test or a Mann-Whitney test was used as appropriate. For imbalance in baseline characteristics between the 2 groups, a log-binomial regression model was fitted adjusting for the variables for which there were important baseline differences. The primary outcome was compared in all patients who triggered the sepsis pathway and in the subgroup identified as having sepsis. To detect a difference in antibiotic appropriateness at 48 hours between $50 \%$ in the control group and $80 \%$ in the intervention group with an $\alpha$ of 0.05 and $80 \%$ power, 45 patients were required in each arm of the study. Due to limited data from the literature and our small pilot study to guide sample size calculations, 8 further controls were enrolled. Data were analyzed using Stata version 14.0 software (StataCorp, College Station, TX).

\section{Ethics}

The study was approved by the South Eastern Local Health District's Human Research Ethics Committee (HREC 15/039).

\section{RES ULTS}

\section{Participants and Baseline Characteristics}

During the 15-week study period, there were 164 referrals for inpatients who triggered the sepsis pathway. Of these, 111 were captured by the IDAT intervention and 53 patients were included as controls (Figure 1). Overall, 5 patients were excluded in the intervention arm and 1 patient was excluded in the control arm because they had previously triggered the sepsis pathway, leaving 106 patients in the intervention arm and 52 patients in the control group for analysis. Among them, 6 patients were excluded from appropriateness analysis due to unassessable baseline appropriateness (Figure 1).

Notable baseline differences between the intervention and control group were prior antibiotic and day of the week trigger (ie, Friday or Saturday) (Table 1). No advanced-care directives were in place that limited antibiotic management.

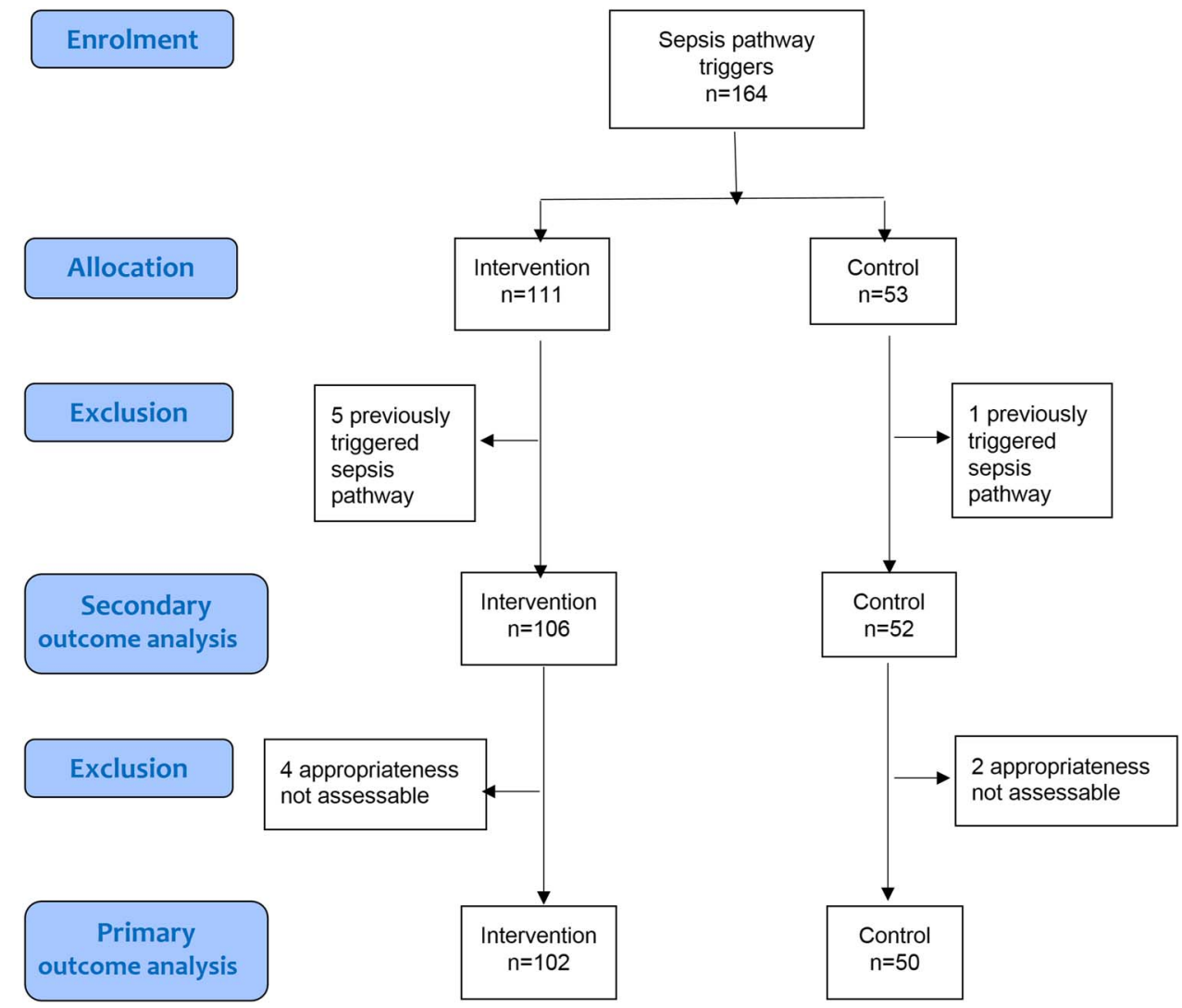

FIGURE 1. Schematic flow diagram of inpatient sepsis pathway study enrollment and analysis. 
тав

\begin{tabular}{|c|c|c|c|}
\hline Baseline Characteristics & $\begin{array}{c}\text { Intervention } \\
\mathrm{N}=106(\%)\end{array}$ & $\begin{array}{c}\text { Control } \\
\mathrm{N}=52(\%)\end{array}$ & $P$ Value $^{\mathrm{a}}$ \\
\hline Female & $55(52)$ & $21(40)$ & .17 \\
\hline Age, $y$, median ( $1^{\text {st }}$ and $3^{\text {rd }}$ quartiles $)$ & $70.5(64.7,82.4)$ & $71.7(64.0-84.4)$ & .99 \\
\hline Mean APACHE II score (SD) & $16.2(6.9)$ & $16.7(6.5)$ & .68 \\
\hline Positive blood culture & $23(22)$ & $9(17)$ & .52 \\
\hline Blood cultures taken at trigger time & $98(92)$ & $46(88)$ & .41 \\
\hline Lactate taken at trigger time & $46(43)$ & $30(58)$ & .09 \\
\hline Source of presumed sepsis & & & .82 \\
\hline Respiratory & $32(30)$ & $15(29)$ & \\
\hline Urinary & $22(21)$ & $11(21)$ & \\
\hline Biliary or gastrointestinal & $18(17)$ & $7(13)$ & \\
\hline Neutropenic & $8(8)$ & $4(8)$ & \\
\hline Skin or surgical site & $6(6)$ & $3(6)$ & \\
\hline Gynecological or genital & $4(4)$ & $0(0)$ & \\
\hline Orthopedic & $1(1)$ & $0(0)$ & \\
\hline Unknown & $15(14)$ & $12(23)$ & \\
\hline Sepsis ${ }^{\mathrm{b}}$ & $61(58)$ & $30(58)$ & .99 \\
\hline Severe sepsis ${ }^{c}$ & $9(8)$ & $6(12)$ & .54 \\
\hline Antibiotics prescribed before trigger & $75(71)$ & $26(50)$ & .044 \\
\hline Trigger on Friday or Saturday & $15(14)$ & $21(40)$ & $<.01$ \\
\hline Initial antibiotic appropriateness ${ }^{\mathrm{d}}$ & $52(51)$ & $28(56)$ & .56 \\
\hline Optimal & $21(21)$ & $8(16)$ & \\
\hline Adequate & $31(30)$ & $19(38)$ & \\
\hline Suboptimal & $35(34)$ & $21(42)$ & \\
\hline Inadequate & $15(15)$ & $2(4)$ & \\
\hline
\end{tabular}

NOTE. APACHE II, Acute Physiology and Chronic Health Evaluation II; SD, standard deviation.

${ }^{a} P$ value from test for difference between intervention and control baseline characteristics.

${ }^{b}$ Defined as infection with 2 or more systemic inflammatory response syndrome (SIRS) criteria ${ }^{6}$ and is inclusive of severe sepsis

${ }^{c}$ Defined as sepsis with organ dysfunction ${ }^{6}$

${ }^{\mathrm{d}}$ Immediate postsepsis trigger appropriateness (adequate + optimal) according to NAPS criteria, ${ }^{10}$ determined in those assessable for appropriateness (intervention $n=102$; controls $n=50$ ).

\section{Antibiotic Appropriateness}

Antibiotic appropriateness at the time of the sepsis pathway trigger was assessable in 152 of 158 patients and was found to be appropriate in 80 of $152(53 \%)$ and inappropriate for 72 of $152(47 \%)$. There was no difference in overall initial antibiotic appropriateness between the intervention and the control group (Table 1). At 48 hours after sepsis pathway trigger, antibiotic management was appropriate in 97 of $102(95 \%)$ intervention patients compared to 38 of $50(76 \%)$ controls (relative risk $[\mathrm{RR}], 1.25$; 95\% confidence interval [CI], 1.06$1.47 ; P<.01)$. The difference remained after adjusting for baseline differences in the day of the week of the trigger (ie, Friday or Saturday versus another day of the week) and pretrigger antibiotics (adjusted RR, 1.24; 95\% CI, 1.04-1.47; $P=.035$ ). There was no difference in 48 -hour appropriateness when analysis was restricted to those with a diagnosis of sepsis. In this subanalysis, 60 of $61(98 \%)$ and 27 of $30(90 \%)$ received appropriate antibiotics at 48 hours in the intervention and control groups, respectively $(P=.09)$. The interrater agreement between 3 ID authors for assessment of appropriateness was $95 \%$.

\section{Sepsis Diagnosis}

Overall, 61 of 106 patients in the intervention group (58\%) and 30 of 52 controls (58\%) had a diagnosis of sepsis. Severe sepsis was present in $8 \%$ of intervention patients compared to $12 \%$ of controls $(P=.54)$. Diagnostic test sampling did not differ markedly; blood cultures were collected in $90 \%$ and serum lactate in $48 \%$ of patients (Table 1).

\section{Intervention Recommendations and Concordance}

In the intervention group, 134 IDAT recommendations were made for 94 patients, among whom 125 (93\%) were followed or partially followed (Table 2). IDAT recommendations included cessation or de-escalation of antibiotics in 56 of 106 patients (53\%) and increasing antibiotic spectrum in 19 of 106 patients $(18 \%)$. Diagnostic investigations were recommended 
TABLE 2. Compliance with Infectious Diseases Advanced Trainee (IDAT) Recommendations in the Intervention Group

\begin{tabular}{lcc}
\hline IDAT Recommendation $^{\text {a }}$ & No. Made & No. Followed (\%) \\
\hline Total & 134 & $125(93)$ \\
Cease antibiotic & 36 & $32(89)$ \\
Decrease spectrum & 20 & $18(90)$ \\
Increase spectrum & 19 & $18(94)$ \\
Escalation plan & 10 & $10(100)$ \\
Change to oral antibiotics & 2 & $2(100)$ \\
Change therapy due to safety & 1 & $1(100)$ \\
Change dose & 6 & $6(100)$ \\
Investigation suggested & 40 & $38(95)$ \\
No recommendation needed & 12 & $\cdots$ \\
\hline
\end{tabular}

${ }^{\mathrm{a}}>1$ intervention possible per patient.

table 3. Clinical Outcomes of Patients Who Triggered the Sepsis Pathway

\begin{tabular}{lccc}
\hline & $\begin{array}{c}\text { Intervention } \\
(\mathrm{N}=106),\end{array}$ & $\begin{array}{c}\text { Control } \\
(\mathrm{N}=52),\end{array}$ & \\
Clinical Outcomes & No. $(\%)^{\mathrm{a}}$ & No. $(\%)^{\mathrm{a}}$ & $P$ Value \\
\hline ICU admission & $16(15)$ & $8(15)$ & .96 \\
Formal ID consult & $11(10)$ & $4(8)$ & .59 \\
Mortality & $14(13)$ & $9(17)$ & .49 \\
Mortality if confirmed sepsis & $9(15)$ & $5(17)$ & .52 \\
Length of stay, d, median & $13(7,27)$ & $17.5(10,29)$ & .14 \\
$\quad\left(1^{\text {st }}, 3^{\text {rd }}\right.$ quartiles) & & & \\
Length of therapy, d, median & $9(5,15)$ & $10.5(5,16.5)$ & .34 \\
$\quad\left(1^{\text {st }}, 3^{\text {rd }}\right.$ quartiles) & & &
\end{tabular}

NOTE. ICU, intensive care unit; ID infectious diseases.

${ }^{\mathrm{a}}$ Unless otherwise specified.

in 40 of 106 patients (38\%). Patients without sepsis were more likely to receive a recommendation to cease an antibiotic ( 29 vs $7 ; P<.01$ ) and to have further investigation (14 vs 20 ; $P<.05)$ and were less likely to receive the recommendation 'increase spectrum' (1 vs $19 ; P<.01)$. Prescriptions for ceftriaxone and pipericillin-tazobactam, the most frequently prescribed antibiotics, were reduced in the intervention group from $34 \%$ (46 of 137 ) to $27 \%$ (25 of 94) and were increased in the control patients from $25 \%$ (13 of 52 ) to $44 \%$ (28 of 64 ) at 48 hours.

\section{Clinical Outcomes}

Clinical outcomes were similar in the intervention and control groups (Table 3). Overall, 24 patients (15\%) were admitted to the ICU and 15 patients (9\%) received a formal ID consult. The difference in mortality between the intervention (14 of 106; $13 \%$ ) and control group (9 of 52;17\%) was not statistically significant $(P=.49)$. The median LOS was 18 days in the control group and 13 days in the intervention group $(P=.14)$. Overall median duration of antibiotic therapy was 9 days for the intervention patients compared to 10.5 days for controls $(P=.34)$.

\section{I S C US S I O N}

This study demonstrates that appropriateness of antibiotic prescribing at 48 hours for non-ICU inpatients triggering the sepsis pathway was significantly improved by early IDAT review. This finding is consistent with other findings that ID intervention improves appropriate antibiotic prescribing in acute care. ${ }^{12-14}$ In the patient subgroup with confirmed sepsis, the rate of appropriate antibiotic therapy in patients at 48 hours were comparable, suggesting that the greatest impact from IDAT intervention was on antibiotic management of those patients triggering the pathway who did not have sepsis. This highlights the importance of monitoring quality sepsis interventions for unintended consequences and a role for AMS.

Initial antibiotic management was inappropriate in almost half of the study population. Optimization was predominantly achieved through de-escalation; an increase in spectrum was the next most frequent recommendation. Suboptimal initial antibiotic prescribing may be due to a combination of poor pathway specificity, overattribution of the diagnosis of sepsis in deteriorating patients, and protocol-driven time pressure to administer antibiotics within 60 minutes for suspected sepsis. Similar rates of hospital inpatient infection misdiagnosis were found to be strongly associated with inappropriate antibiotic use. ${ }^{15}$

The relatively high rate of blood culture sampling suggests effective junior doctor sepsis pathway process training. However, protocolizing inpatient sepsis antibiotic management is difficult given that many patients may already be receiving therapy. Junior doctors (ie, with the least experience) are typically required to make acute inpatient assessments and management decisions, often with less on-site senior medical support compared with emergency departments and ICUs. The imperative for early antibiotic initiation in sepsis management pathways exacerbates this scenario. While appropriate antibiotic therapy should be commenced as early as possible and within 60 minutes for severe sepsis and septic shock, ${ }^{3}$ there is little evidence demonstrating the benefit of early antibiotic administration in uncomplicated sepsis. ${ }^{4,5,17}$ Thus, it may be more judicious for sepsis pathways to focus on early antibiotic delivery ( $<60$ minutes) for cases suspected of severe sepsis and septic shock, while further timely investigation should be undertaken for cases suspected of uncomplicated sepsis.

Antibiotic review and rationalization post sepsis trigger is recommended in sepsis pathways, ${ }^{7}$ but in clinical practice, these may not be prioritized. Despite a comprehensive hospitalwide AMS program ${ }^{16}$ and intensive sepsis pathway preimplementation preparation, IDAT intervention facilitated a further significant sizeable improvement in antibiotic prescribing over standard inpatient care beyond the sepsis trigger. Inadequate de-escalation and overprescription of broad-spectrum antibiotics may represent avoidable harm and is important to monitor antibiotic administration in sepsis interventions. AMS efforts could be integrated with sepsis pathway protocols to optimize appropriate initial and ongoing 
antibiotic therapies. Appropriate, guideline-adherent empirical antibiotic management has been shown to be associated with a relative risk reduction for mortality of $35 \%$ (relative risk, 0.65 ; 95\% CI, 0.54-0.80; $P<.0001){ }^{18}$

Secondary outcomes including length of therapy, LOS, ICU admission, formal ID consultation, and mortality were not significantly different between the intervention and control groups. However, this study was not powered to detect a statistically significant difference in clinical outcomes. These clinical outcomes were used to detect potential harms arising from the intervention. Overall mortality of $15 \%$ is consistent with other reported inpatient sepsis mortality $(15 \%-35 \%)^{19,20}$ and was not different among patients diagnosed with sepsis. Although there was no difference in LOS, emerging evidence suggests that improving antibiotic appropriateness ${ }^{8,21}$ and ID specialist review ${ }^{13,14}$ may impact this outcome in a larger study.

This study had several limitations including study size, duration, generalizability, and selection bias. Although the study population was small, it was adequately powered to detect the primary outcome of a significant difference in antibiotic appropriateness in patients 48 hours after triggering the sepsis pathway. Standardized national criteria for antibiotic appropriateness ${ }^{10}$ were utilized in this study; however, observer bias was a potential risk. This was addressed by using additional blinded assessments by 2 senior ID coauthors, which demonstrated high interrater agreement.

The study intervention was a brief case review by 1 IDAT in a single institution. Therefore, benefits may be operator dependent and are not necessarily generalizable. PDSA cycles may generate different interventions in other settings. Additionally, the capacity of both AMS and quality programs depend on the institution's resources. To remain cost neutral, additional time required for the IDAT intervention during this study was taken from routine AMS responsibilities. Inclusion of early IDAT sepsis review within AMS programs may prove to be an efficient use of AMS resources, particularly if it is prioritized for higher-acuity patients.

This study was not randomized; therefore, selection bias is a limitation. The small sample size restricts the number of potential baseline differences that can be adjusted for. Nevertheless, those differences considered most likely to influence group allocation and appropriateness, prior antibiotics and triggering the pathway on a Friday or Saturday, were also statistically significant. After adjusting for these, the significance of the impact of IDAT intervention on antibiotic appropriateness remained. Nevertheless, the need to ensure ongoing optimal antibiotic management 'after hours' is highlighted by this study and other research demonstrating an association between weekend day admissions and adverse clinical outcomes. ${ }^{22}$ Other baseline differences were unlikely to be clinically significant for the primary study end point, antibiotic appropriateness.

The difficulty with achieving diagnostic specificity in sepsis is acknowledged in the recent change in the International Consensus Definitions for Sepsis and Septic Shock (SEPSIS-3). ${ }^{23}$ New sepsis definitions published after the completion of this study hone the criteria for sepsis toward organ dysfunction as a manifestation of an injurious host response to infection rather than focusing on systemic inflammation. Application of SEPSIS-3 criteria in this study would have improved specificity and therefore may have reduced unnecessary exposure to broad-spectrum antibiotics.

In Australia, improvement in emergency department sepsis recognition and care followed implementation of the CEC emergency department sepsis pathway in $2011 .{ }^{19}$ However, a significant rise in non-ICU inpatient sepsis mortality was also observed, the cause of which was unclear. ${ }^{19}$ Benefits of adult inpatient sepsis pathways have yet to be established. Further CEC inpatient sepsis pathway revision has occurred since this study was conducted. ${ }^{24}$ An evaluation of the impact of these pathways on much needed improvement in inpatient sepsis recognition and management is highly anticipated.

In conclusion, hospital inpatient sepsis remains a clinical challenge. Lack of sepsis diagnostic specificity hinders clinical sepsis pathway implementation and may drive inappropriate antibiotic use. Infectious disease AMS significantly improved antibiotic appropriateness for non-ICU inpatients with suspected sepsis, with the greatest impact on those patients who were misdiagnosed. Hospitals implementing sepsis pathways should evaluate their diagnostic specificity and patients' antibiotic exposure and consider how AMS may optimize these. Larger prospective studies are needed to validate these findings and evaluate clinical outcomes.

\section{ACKNOWLEDGMENTS}

Financial support: No financial support was provided relevant to this article.

Potential conflicts of interest: All authors report no conflicts of interest relevant to this article.

Address correspondence to Pamela Konecny, St George Hospital, Department of Infectious Diseases, Immunology \& Sexual Health, 2 South St, Kogarah, Sydney, NSW 2217, Australia (pam.konecny@health.nsw.gov.au).

\section{REFERENCES}

1. Cohen J, Vincent J-L, Adhikari NKJ, et al. Sepsis: a roadmap for future research. Lancet Infect Dis 2015;15:581-614.

2. Vincent J, Marshall JC, Silvio AN, et al. Assessment of the worldwide burden of critical illness: the Intensive Care Over Nations (ICON) audit. Lancet Respir Med 2014;2:380-386.

3. Kumar A, Roberts D, Wood KE, et al. Duration of hypotension before initiation of effective antimicrobial therapy is the critical determinant of survival in human septic shock. Crit Care Med 2006;34:1589-1596.

4. Sterling SA, Miller WR, Pryor J, et al. The impact of timing of antibiotics on outcomes in severe sepsis and septic shock: a systematic review and meta-analysis. Crit Care Med 2015;43: 1907-1905.

5. Ryoo SM, Kim WY, Sohn CH, et al. Prognostic value of timing of antibiotic administration in patients with septic shock treated with early quantitative resuscitation. Am J Med Sci 2015;349: 328-333. 
6. Dellinger RP, Levy MM, Rhodes A, et al. Surviving sepsis campaign: international guidelines for management of severe sepsis and septic shock: 2012. Crit Care Med 2013;41: 580-637.

7. Sepsis toolkit: inpatient program implementation guide. Clinical Excellence Commission website. http://www.cec.health. nsw.gov.au/_data/assets/pdf_file/0010/276067/Sepsis_Toolkit_ inpatient-full.pdf. Published 2014. Accessed June 1, 2017.

8. Davey $\mathrm{P}$, Brown E, Charani E, et al. Interventions to improve antibiotic prescribing practices for hospital inpatients. Cochrane Database Syst Rev 2013;4. doi: 10.1002/14651858.

9. Taylor MJ, McNicholas C, Nicolay C, et al. Systematic review of the application of the plan-do-study-act method to improve quality in healthcare. BMJ Quality \& Safety 2013:1-9.

10. Antimicrobial prescribing practice in Australian hospitals: results of the 2014 National Antimicrobial Prescribing Survey. Australian Commission on Safety and Quality in Health Care website. https://www.safetyandquality.gov.au/wp-content/uploads/ 2016/03/Antimicrobial-prescribing-practice-in-Aust-hospitalsNAPS-2014-Results.pdf. Published 2016. Accessed June 1, 2017.

11. Therapeutic Guidelines Limited website. http://www.tg.org.au. Published 2015. Accessed June 1, 2017.

12. Pulcini C, Botelho-Nevers E, Dyar OJ, Harbarth S. The impact of infectious disease specialists on antibiotic prescribing in hospitals. Clin Microbiol Infect 2014;20:963-972.

13. Butt AA, Al Kaabi N, Saifuddin M, et al. Impact of infectious diseases team consultation on antimicrobial use, length of stay and mortality. Am J Med Sci 2015;350:191-194.

14. Fariñas M, Saravia G, Calvo-Montes J, et al. Adherence to recommendations by infectious disease consultants and its influence on outcomes of intravenous antibiotic-treated hospitalized patients. BMC Infect Dis 2012;12:292.
15. Filice GA, Drekonja DM, Thurn JR, Hamann GM, Masoud BT, Johnson JR. Diagnostic errors that lead to inappropriate antimicrobial use. Infect Control Hosp Epidemiol 2015;36:949-956.

16. Bond SE, Chubaty AJ, Adhikari S, et al. Outcomes of multisite antimicrobial stewardship program implementation with a shared clinical decision support system. J Antimicrob Chemother 2017. doi: 10.1093/jac/dkx080.

17. Wisom A, Eaton V, Gordon D, et al. INITIAT-E.D.: impact of timing of initiation of antibiotic therapy on mortality of patients presenting to an emergency department with sepsis. Emergency Medicine Australasia 2015;27:196-207.

18. Schuts EC, Hulscher MEJL, Mouton JW, et al. Current evidence on hospital antimicrobial stewardship objectives: a systematic review and meta-analysis. Lancet Infect Dis 2016;16:847-856.

19. Burrell AR, McLaws M-L, Fullick M, et al. Sepsis kills: early intervention saves lives. Med J Aust 2016;204:1.e1-e7.

20. Kaukonen K, Bailey M, Suzuki S, et al. Mortality related to severe sepsis and septic shock among critically ill patients in Australia and New Zealand, 2000-2012. JAMA 2014;311:1308-1316.

21. Yokota PK, Alexandre RM, Marines DV, et al. Impact of appropriate antimicrobial therapy for patients with severe sepsis and septic shock: a quality improvement study. PLoSOne 2014;9:e104475.

22. Fremantle N, Ray D, McNulty D, et al. Increased mortality associated with weekend hospital admission: a case for expanded seven day services? BMJ 2015;351:h4596.

23. Singer M, Deutschman CS, Warren Seymour C, et al. The third international consensus definitions for sepsis and septic shock (Sepsis-3). JAMA 2016;315:801-810.

24. Sepsis kills. Sepsis tools. Clinical Excellence Commission website. http://www.cec.health.nsw.gov.au/_data/assets/pdf_file/ 0005/299417/adult-antibiotic-guideline-severe-sepsis-septic-shocksept2016.pdf. Published 2016. Accessed June 1, 2017. 